\title{
Multiple roles of the epithelium-specific ETS transcription factor, ESE-1, in development and disease
}

Jordan R Oliver ${ }^{1,2}$, Rahul Kushwah ${ }^{1,2}$ and Jim $\mathrm{Hu}^{1,2}$

The E26 transformation-specific (ETS) family of transcription factors comprises of 27 and 26 members in humans and mice, respectively, which are known to regulate many different biological processes, including cell proliferation, cell differentiation, embryonic development, neoplasia, hematopoiesis, angiogenesis, and inflammation. The epitheliumspecific ETS transcription factor-1 (ESE-1) is a physiologically important ETS transcription factor, which has been shown to play a role in the pathogenesis of various diseases, and was originally characterized as having an epithelial-restricted expression pattern, thus placing it within the epithelium-specific ETS subfamily. Despite a large body of published work on ETS biology, much remains to be learned about the precise functions of ESE-1 and other epithelium-specific ETS factors in regulating diverse disease processes. Clues as to the specific function of ESE-1 in the setting of various diseases can be obtained from studies aimed at examining the expression of putative target genes regulated by ESE- 1 . Thus, this review will focus primarily on the various roles of ESE-1 in different pathophysiological processes, including regulation of epithelial cell differentiation during both intestinal development and lung regeneration; regulation of dendritic cell-driven T-cell differentiation during allergic airway inflammation; regulation of mammary gland development and breast cancer; and regulation of the effects of inflammatory stimuli within the setting of synovial joint and vascular inflammation. Understanding the exact mechanisms by which ESE-1 regulates these processes can have important implications for the treatment of a wide range of diseases.

Laboratory Investigation (2012) 92, 320-330; doi:10.1038/labinvest.2011.186; published online 12 December 2011

KEYWORDS: cancer; development; epithelial cell differentiation; ESE-1; ETS transcription factor; inflammation; regeneration

The E26 transformation-specific (ETS) family of transcription factors is characterized by a highly conserved 84 -aminoacid DNA-binding domain, known as the ETS domain. ${ }^{1}$ Because the first member of the ETS family, the v-ets oncogene, was originally discovered as part of a fusion protein with gag and myb expressed by the E26 avian erythroblastosis-transforming retrovirus and its DNA-binding domain is E26 transformation-specific, this 84-amino-acid DNA-binding domain was named the ETS domain. ${ }^{1}$ The ETS domain is usually located within the carboxyl-terminal region of the protein as a winged helix-turn-helix structural motif and mediates binding to sites of purine-rich DNA, commonly containing a core consensus sequence of GGAA/T, within the promoter and enhancer regions of target genes. ${ }^{2,3}$ Many ETS transcription factors also contain a pointed domain, which is located within the amino-terminal region and is involved in protein-protein interactions. ${ }^{2}$ Approxi- mately 30 members of the ETS transcription factor family have been identified in mammals (ie 27 in humans and 26 in mice) ${ }^{4}$ and have been shown to play crucial roles in the regulation of many physiological and pathological processes, such as embryonic development, ${ }^{5,6}$ neoplasia, ${ }^{6-9}$ hematopoiesis, ${ }^{10,11}$ angiogenesis, ${ }^{12-14}$ and inflammation. ${ }^{15}$

Although many ETS family members are expressed in non-epithelial cells, such as hematopoietic and endothelial cells, epithelium-specific ETS transcription factor-1 (ESE-1) belongs to the ESE subfamily of ETS transcription factors, which are believed to be expressed only in epithelial-rich tissues, such as stomach, small intestine, colon, pancreas, trachea, lung, kidney, salivary gland, prostate gland, mammary gland, uterus, and skin. ${ }^{16,17}$ Since its initial discovery and characterization, ESE-1 has been designated by many other names, such as E74-like transcription factor-3 (Elf3), ETS-related transcription factor (Ert), epithelial-restricted

\footnotetext{
${ }^{1}$ Department of Laboratory Medicine and Pathobiology, University of Toronto, Toronto, Ontario, Canada and ${ }^{2}$ Physiology and Experimental Medicine Research Program, The Hospital for Sick Children, Toronto, Ontario, Canada

Correspondence: Dr J Hu, PhD, Physiology and Experimental Medicine Research Program, The Hospital for Sick Children, 555 University Avenue, Toronto, Ontario, Canada M5G1X8.

E-mail: jim.hu@utoronto.ca

Received 25 August 2011; revised 27 October 2011; accepted 29 October 2011
} 
with serine box (Esx) and Jen. Elf3 is the murine homolog for the human ESE-1 gene, and is $89 \%$ identical to its human homolog at the amino-acid level. ${ }^{16}$ Northern blot analysis of ESE-1 mRNA expression in human tissues has previously shown the highest levels of expression to occur in the small intestine, colon, and uterus. ${ }^{16,17}$ Very little to no expression of ESE-1 was detected in many epithelial-poor tissues, such as the spleen, thymus, brain, heart, and skeletal muscle. ${ }^{16,17}$ Similar patterns of expression for Elf3 have also been demonstrated in mouse tissues. ${ }^{16}$ Expression of ESE-1 has also been detected in prostate, colon, breast, and cervical cancerderived cell lines. ${ }^{18}$ In addition to containing both an ETS domain and a pointed domain, ESE-1 also contains both an $\mathrm{A} / \mathrm{T}$ hook domain, which is commonly found in highmobility group (HMG) proteins and various other nuclear factors, ${ }^{17}$ and a PEST domain (rich in proline, glutamic acid, serine, and threonine amino acids), which is commonly found in rapidly degraded proteins. ${ }^{16}$ While the A/T hook domain has been shown to mediate binding to the minor groove of AT-rich tracts of double-stranded DNA, ${ }^{17}$ the PEST domain is believed to play a role in protein stability owing to protease targeting. ${ }^{16}$ Interestingly, ESE-1 also contains a serine- and aspartic acid-rich (SAR) domain, ${ }^{19}$ in which a p21activated kinase-1 (Pak1) phosphorylation site is present. ${ }^{20}$

Other members of the ESE subfamily of ETS factors also include ESE-2 (also known as Elf5), ESE-3 (also known as Ehf), and prostate-derived ETS transcription factor (PDEF). The retained homology of the ETS domains and the core consensus sequence for the preferred DNA binding site for each ESE subfamily member are illustrated in Figures 1 and 2, respectively. Although the DNA-binding domains of ESE-1 and ESE-2 are closely related with 65\% homology within their respective ETS domains, ESE-2 appears to have a more limited and restricted DNA binding specificity than ESE- $1{ }^{21,22}$ More specifically, whereas ESE- 2 can only interact with DNA sequences that contain a GGAA core, ESE-1 also expresses affinity for GGAT in addition to GGAA. ${ }^{21,22}$ The ETS domain of ESE-3 is 84 and 65\% identical to the ETS domains of ESE-1 and ESE-2, respectively. ${ }^{23}$ In addition, although ESE-3 prefers binding to DNA sequences that contain a GGAA core, it does have some affinity for GGAT. PDEF appears to be more divergent from ESE-1, ESE-2, and
ESE-3 with only 42 , 45, and 43\% amino-acid identity within their respective ETS domains. Importantly, it should be acknowledged that PDEF uniquely prefers binding to DNA sequences that contain a GGAT rather than a GGAA core. ${ }^{24}$

In comparison to ESE-1, the tissue expression pattern for ESE-2 is even more restricted. ${ }^{21,22}$ More specifically, whereas ESE-1 is expressed in the majority of epithelial cell types, ESE-2 expression is restricted to differentiated keratinocytes within the epidermis of the skin and glandular epithelium, such as salivary gland, prostate gland, mammary gland, kidney, lung, and stomach. ${ }^{21,22}$ In addition, expression of ESE-2 is absent within the small intestine and colon, ${ }^{21,22}$ which are tissues in which ESE-1 is highly expressed. ${ }^{16,17}$ Although ESE-2 does not have an additional DNA-binding domain, such as the HMG-A/T hook domain that is found in ESE-1, it does have an amino-terminal negative regulatory domain that inhibits DNA binding and is not present in ESE-1 or ESE-3. ${ }^{21,22}$ In similarity to ESE-1 and ESE-2, ESE-3 is exclusively expressed in a subset of epithelial cells with highest expression in glandular epithelium, such as prostate gland, pancreas, salivary gland, and trachea. ${ }^{23}$ In addition, low expression levels of ESE-3 have previously been detected in the mammary gland, lung, kidney, and colon, and

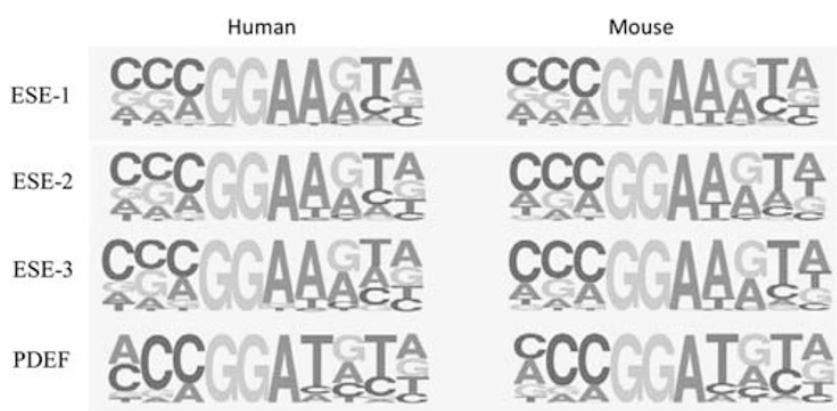

Figure 2 Schematic representation of the respective consensus sequence for the preferred DNA binding site (ie ETS-binding profiles) for both human and mouse epithelium-specific ETS transcription factor-1 (ESE-1), ESE-2, ESE-3, and prostate-derived ETS transcription factor (PDEF), as determined using microwell-based transcription factor-DNA-binding assays. The height of a letter at a particular position is directly proportional to the effect of that nucleotide on the binding affinity. This figure was recreated with permission from a previously published manuscript (see Wei et $a l^{3}$ ).

\section{ESE-1 273 THLWEFIRDILIHPELNEGLMKWENRHEGVFKFLRSEAVAQLWGQKKKNSNMTY ESE-2 173 SHLWEFVRDLLLSPEENCGILEWEDREQGIFRVVKSEALAKMWGQRKKNDRMTY \\ ESE-3 207 THLWEFIRDILLNPDKNPGLIKWEDRSEGVFRFLKSEAVAQLWGKKKNNSSMTY PDEF 249 IHLWQFLKELLLKPHSYGREIRWLNKEKGIFKIEDSAQVARLWGIRKNRPAMNY \\ ESE-1 328 EKLSRAMRYYYKREILERVD-GRRLVYKFGKNSSGWKEEEVLQSRN \\ ESE-2 228 EKLSRALRYYYKTGILERVD--RRLVYKFGKNAHGWQEDKL \\ ESE-3 262 EKLSRAMRYYYKREILERVD-GRRLVYKFGKNARGWRENEN \\ PDEF 304 DKLSRSIRQYYKKGIIRKPDISQRLVYQFVHPI}

Figure 1 Alignment of the protein sequences for the highly conserved E26 transformation-specific (ETS) (DNA-binding) domain of the human versions of epithelium-specific ETS transcription factor-1 (ESE-1), ESE-2, ESE-3, and PDEF. The ETS domain is located at the carboxyl-terminus of all four proteins. The number at the beginning of each line indicates the amino-acid position within the protein. The terminal amino-acid sequence outside the ETS domain is underlined. 
absolutely no expression has been detected in the small intestine. ${ }^{23}$ Interestingly, PDEF is almost exclusively expressed in the epithelial layers of the prostate gland with low levels of expression also detected in ovary as well as in mammary and salivary gland, which are all hormone-regulated epithelial tissues. ${ }^{24}$ It is also worth noting that no expression of PDEF has been detected in certain epithelialrich tissues, such as the gastrointestinal tract, kidney, or lung. ${ }^{24}$ Similar to ESE-1, PDEF also contains two putative PEST domains. ${ }^{24}$ Several potential phosphorylation sites are also present within the ESE transcription factors, including tyrosine kinase sites, protein kinase $\mathrm{C}$ sites, casein kinase II sites, and c-Jun $\mathrm{NH}_{2}$-terminal kinase/p38/extracellular signal-regulated kinase kinase sites. ${ }^{21-24}$ Moreover, in vitro transient transfection studies using multiple ETSresponsive reporter gene constructs have demonstrated that ESE factors can function both as transcriptional activators and repressors. ${ }^{18}$

This review will focus on the various roles of the epithelium-specific ETS transcription factor, ESE-1, in different pathophysiological processes within epithelial-rich tissues. In particular, the involvement of ESE-1 in regulating many of these processes is based on findings obtained from in vivo studies utilizing mice with a null mutation of Elf3 as well as in vitro studies utilizing various primary cells and cell lines of epithelial origin. In contrast to previous belief, ESE-1 is not expressed exclusively in epithelial cells and numerous studies have shown an induction of ESE-1 in cells of non-epithelial origin in response to inflammatory stimuli. Therefore, various roles of ESE-1 in relevant inflammatory diseases will also be discussed in this review. In addition, the putative target genes regulated by ESE-1 are listed in Table 1, and will be discussed in further detail in the following sections with regard to how expression of each target gene is modulated by ESE- 1 and in which tissue or cell type it has been shown to occur.

\section{ESE-1 IN EARLY EMBRYONIC DEVELOPMENT}

Mice with a null mutation of Elf3 have previously been generated through targeted gene disruption, and on average, approximately $30 \%$ of the resultant homozygous mutant mice die in utero at around embryonic day $11.5,{ }^{25}$ suggesting that Elf3 may potentially play an important role in embryonic development. Indeed, others have also reported an involvement of Elf3 and other ETS transcription factors in regulating mouse pre-implantation embryonic development. ${ }^{5}$ Interestingly, evidence of abnormalities in the endometrium of the uterus characterized by disorganization of the columnar epithelium and decreased number of uterine glands has been observed in surviving Elf3-deficient (Elf3-/-) mice, ${ }^{25}$ which may in part also be related to the partial embryonic fatality of the Elf3-/- offspring in utero. However, it is important to point out that the exact cause(s) of the partial embryonic lethality of Elf3-/- mice during gestation is unknown currently. ${ }^{25}$ Future studies aimed at elucidating the exact role of ESE-1 in early embryonic development could potentially provide new insight for this epithelial-specific transcription factor in regulating various differentiation pathways. For instance, experiments focused on examining embryonic stem cell differentiation to endoderm, and then subsequently to other epithelial cell lineages ${ }^{26,27}$ in Elf3-/- mice as well as utilizing the inducible pluripotent stem (iPS) cell strategy ${ }^{28,29}$ of genetically reprogramming somatic cells (eg fibroblasts) isolated from Elf3-/ - mice into pluripotent embryonic stem cell-like cells or iPS cells can potentially reveal a direct involvement of ESE-1 in regulating cellular differentiation pathways during embryonic development.

\section{ESE-1 IN REGULATING EPITHELIAL CELL DIFFERENTIATION DURING INTESTINAL DEVELOPMENT}

Many of the Elf3-/- progeny that survive until birth have been observed to have diminished weight gain and eventually develop a 'wasting syndrome' that is characterized by a malnourished physical appearance, watery diarrhea, and lethargy. ${ }^{25}$ Most importantly, Elf3-/- mice also exhibit a distinct phenotype in the small intestine during fetal/neonatal development, which includes severe morphological alterations in tissue architecture manifested by poor villus formation along with improper morphogenesis of the microvilli and defective terminal differentiation of absorptive enterocytes and mucus-secreting goblet cells. ${ }^{25}$ Moreover, it has been shown that the enterocytes within the small intestinal epithelium of Elf3-/- mice express reduced protein levels of transforming growth factor- $\beta$ type II receptor (TGF- $\beta$ RII), which is a potent inhibitor of cell proliferation and an inducer of epithelial cell differentiation. ${ }^{25}$ It was subsequently demonstrated that ectopic expression of the human TGF- $\beta$ RII transgene specifically in the intestinal epithelium of Elf3-/- mice could rescue the previously characterized intestinal defects of Elf3- - mice. ${ }^{30}$ Thus, this phenotypic rescue had provided strong in vivo evidence that Elf3 is the critical upstream regulator of TGF- $\beta$ RII gene expression in the mouse small intestinal epithelium. ${ }^{30}$ Indeed, many in vitro studies have also established that the TGF- $\beta$ RII gene is a definite target of Elf3, and that Elf3 transactivates the TGF- $\beta$ RII gene promoter by binding to two adjacent ETS binding sites. ${ }^{31-37}$ Interestingly, human gastric cancer cell lines do not express ESE-1 mRNA and in turn show undetectable levels of TGF- $\beta$ RII mRNA. ${ }^{38}$ Further evidence of ESE-1-mediating TGF- $\beta$ RII gene expression has also been provided from experiments with human colon cancer cell lines. ${ }^{34}$ In human colonic epithelial cells, ESE-1 has also been shown to regulate gene expression of the proinflammatory cytokine, macrophage inflammatory protein-3 $\alpha$ (MIP-3 $\alpha){ }^{39}$

Another group has previously reported that CR6-interacting factor 1 (Crif1) plays an essential role in Elf3-mediated intestinal development by functioning as a transcriptional co-activator of Elf3 during terminal differentiation of the 
Table 1 List of putative target genes regulated by ESE-1

Target gene

Transforming growth factor- $\beta$ type $/ /$ receptor (TGF- $\beta$ RII) $\uparrow$

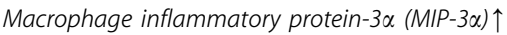

Small proline-rich protein $1 B(S P R R 1 B) \uparrow$

Epithelium-specific ETS transcription factor-3 (ESE-3) $\uparrow$

Interleukin-6 (IL-6)

Interleukin-12 (IL-12)

Lysozyme $(L Y Z) \uparrow$

Whey acidic protein (WAP) $\uparrow$

Erythroblastic leukemia viral oncogene homolog 2 (ErbB-2) $\uparrow$

Small proline-rich protein $2 A(S P R R 2 A) \uparrow$

Transglutaminase-3 (TGM-3) $\uparrow$

Profilaggrin $\uparrow$

Keratin $4(K 4) \downarrow$
Tissue/cell type in which ESE-1 regulates target gene

References

Mouse intestinal epithelium

Mouse bronchiolar airway epithelium

Mouse embryonal carcinoma cell line (F9)

Human embryonic kidney cell line (HEK 293T)

$33,35,37$

Human hepatoblastoma cell line (HepG2)

$33,35,37$

Human gastric cancer cell line (SNU-620)

Human colon cancer cell line (RKO)

Human breast cancer cell lines (SK-BR3, Hs578t)

Human cervical cancer cell line (HeLa 229)

Human colonic epithelial cell line (Caco-2)

Primary human tracheobronchial epithelial cells

Human bronchial epithelial cell line (BEAS-2B)

Human lung adenocarcinoma epithelial cell line ( $\mathrm{NCl}-\mathrm{H} 441)$

Human bronchial epithelial cell line (BEAS-2B)

Human lung carcinoma cell line (A549)

Mouse lung tissue

Primary mouse airway epithelial cells

Primary mouse bone marrow-derived dendritic cells

Human bronchial epithelial cell line (BEAS-2B)

Primary mouse bone marrow-derived dendritic cells

Primary human bronchial airway epithelial cells

Human lung carcinoma cell line (A549)

Human lung mucoepidermoid carcinoma-derived cell line ( $\mathrm{NCl}-\mathrm{H} 292)$

Human lung adenocarcinoma epithelial cell line ( $\mathrm{NCl}-\mathrm{H} 441)$

Human cervical cancer cell line (HeLa)

Mouse mammary epithelial cell line (HC-11)

Simian kidney fibroblast cell line (COS)

Human cervical cancer cell line (HeLa)

Primary human foreskin keratinocytes

Human keratinocyte cell line ( $\mathrm{HaCaT})$

Simian kidney fibroblast cell line (COS-1)

Mouse embryonic fibroblast cell line (NIH 3T3)

Human esophageal squamous carcinoma cell lines (TE-11, TE-12)

Human cervical cancer cell line (HeLa)

Normal human epidermal keratinocytes (NHEK cells)

Human keratinocyte cell line ( $\mathrm{HaCaT})$

Human cervical cancer cell line (HeLa)

Human neuroblastoma cell line (SK-N-AS)

Normal human epidermal keratinocytes (NHEK cells)

Human keratinocyte cell line (HaCaT)

Human cervical cancer cell line (HeLa)

Human neuroblastoma cell line (SK-N-AS)

Human esophageal squamous carcinoma cell lines (TE-11, TE-12)

Human cervical cancer cell line (HeLa)
25,30

42

31

44

0 37 . 32 
Table 1 Continued

Target gene

Small proline-rich protein $1 A(S P R R 1 A) \uparrow$

Small proline-rich protein $3(S P R R 3) \uparrow$

K12 keratin $\uparrow$

Tissue inhibitor of metalloproteinase 3 (TIMP3) $\uparrow$

Angiopoietin-1 (Ang-1) $\uparrow$

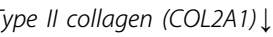

Inducible nitric-oxide synthase (NOS2) $\uparrow$

Cyclooxygenase-2 (COX-2)
Tissue/cell type in which ESE-1 regulates target gene

References

Primary human neonatal foreskin epidermal keratinocytes

62

Primary human neonatal foreskin epidermal keratinocytes

Mouse corneal epithelium

Human corneal epithelial cell line (HCE)

Rat retinal pigment epithelium

Human retinal pigment epithelium cell lines (D407, hTERT-RPE1)

Primary human synovial fibroblasts

Mouse embryonic fibroblast cell line (NIH 3T3)

Human embryonic kidney cell line (HEK 293)

Human breast cancer cell line (MCF-7)

Primary human chondrocytes

Human chondrocyte cell lines (T/C-28a2, C-28/12)

Primary human aortic smooth muscle cells (HASMCs)

Primary rat aortic smooth muscle cells (RASMCs)

Human acute monocytic leukemia cell line (THP-1)

Mouse leukaemic monocyte macrophage cell line (RAW 264.7)

Mouse thoracic aorta smooth muscle and endothelium

Human acute monocytic leukemia cell line (THP-1)

Mouse leukaemic monocyte macrophage cell line (RAW 264.7)

Human chondrocyte cell line (T/C-28a2)

67

$\uparrow$, Expression of target gene is upregulated by ESE-1; $\downarrow$, expression of target gene is downregulated by ESE- 1 .

intestinal epithelium. ${ }^{40}$ The intestinal epithelium-specific Crif1-deficient (Crif1-/-) mice, which were used in this study, died soon after birth and displayed severe alterations in tissue architecture of the developing small intestine, including poor microvillus formation and abnormal differentiation of absorptive enterocytes, and these phenotypes were largely similar to those previously observed in Elf3-/mice. ${ }^{40}$ It was also shown that Crif1 interacted with Elf3 through its ETS DNA-binding domain and enhanced the transcriptional activity of Elf3 by regulating its DNA-binding activity, whereas knockdown of Crif1 by RNA interference conversely attenuated the transcriptional activity of Elf3. ${ }^{40} \mathrm{In}$ addition, the expression level of TGF- $\beta$ RII, a critical target gene of Elf3, was also dramatically reduced in the Crif1-/mice, ${ }^{40}$ thus suggesting that both Elf3 and Crif1 cooperate in regulating transcription of the TGF- $\beta$ RII gene. However, subsequent in vitro experiments aimed at examining the detailed molecular interactions between Elf3 and its potential binding partners at the TGF- $\beta$ RII gene promoter are required to know the exact molecular mechanism of how TGF- $\beta$ RII gene transcription is regulated. In addition, even though we now have a clearer understanding of the molecular mechanisms of Elf3-mediated intestinal development in mice, further studies are needed to examine a potential association of human ESE-1 with related gastrointestinal diseases, such as inflammatory bowel disease and colorectal cancer.

\section{ESE-1 IN LUNG CANCER, DEVELOPMENT, AND REGENERATION}

Expression of ESE-1 has been detected in some human lung cancers, such as large-cell carcinoma and adenocarcinoma, and in lung cancer-derived cell lines, such as A549. ${ }^{16}$ Overexpression of the squamous differentiation marker, small proline-rich protein 1B (SPRR1B), in the bronchial airway epithelium is a marker for early metaplastic changes induced by various toxicants/carcinogens. ${ }^{41}$ Interestingly, it has been shown that induction of SPRR1B gene expression in bronchial epithelial cells is mediated in part by protein-protein interactions between ESE-1 and other transcription factors, such as specificity protein 1 and activator protein 1 (AP-1), at the proximal and distal promoter regions, respectively. ${ }^{41}$ With regards to lung development, very high levels of Elf3 expression have previously been detected within the 
developing fetal mouse lung. ${ }^{16}$ However, further experiments are definitely required to obtain a better understanding of the exact role played by this transcription factor in both lung cancer and development.

In similarity to the pathological and physiological processes of lung cancer and lung development, we have recently found an involvement of Elf3 in the regulation of lung regeneration in adult mice after treatment with the Clara cell-specific toxicant, naphthalene. ${ }^{42}$ More specifically, the kinetics of cell proliferation and mitosis as well as Clara cell renewal was delayed within the distal bronchiolar airway epithelium of Elf3-/- mice as compared with their wildtype counterparts during repair following naphthaleneinduced Clara cell injury. ${ }^{42}$ In contrast, there was a significantly greater level of cell proliferation and mitosis within the peribronchiolar interstitium of Elf3- $/$ - mice than in wild-type mice. ${ }^{42}$ As the absence of Elf3 did not affect the severity of naphthalene-induced Clara cell damage in Elf3- - mice, ${ }^{42}$ the observed differences in airway epithelial repair kinetics between wild-type and Elf3-/- mice were most likely not due to differences in the extent of initial injury. The delayed kinetics of cell proliferation and Clara cell reconstitution observed within the airway epithelium of Elf3-/ - mice was more likely due to changes in gene expression for proteins involved in regulating epithelial cell proliferation and/or differentiation during repair after injury. Indeed, we had also found that Elf3-/- mice express substantially reduced levels of TGF- $\beta$ RII, which is a well-known transcriptional target gene of Elf3 and is involved in the induction of epithelial cell differentiation, in the bronchiolar airway epithelium both basally and during repair after naphthalene injury. ${ }^{42}$ Collectively, these findings suggest that Elf3 plays an important role in the regulation of lung cell proliferation and differentiation during repair of the injured airway epithelium. Furthermore, it is worth noting that as the naphthalene-induced model of acute airway epithelialspecific injury and subsequent repair is not normally associated with excessive inflammation, ${ }^{43}$ it is more likely that Elf3 is contributing to this pathophysiological process through its role as a regulator of epithelial homeostasis rather than as a mediator of inflammation. It must be discussed, however, that a major caveat of the Elf3- $/$ - mouse model utilized in our study is the absence of Elf3 during lung development. Thus, the possibility of compromised lung development in these Elf3-/- mice cannot be ruled out as it has been previously reported that Elf3 is highly expressed in the developing fetal mouse lung. ${ }^{16}$ Therefore, the possibility of an impairment of lung development resulting in a potential reduction of progenitor cells in adult Elf3-/- mice could possibly play a role in the delayed repair kinetics observed in these mice following naphthalene injury. Future studies focused on examining progenitor cells during lung development in Elf3-/- mice as well as airway epithelial repair after conditionally ablating Elf3 in adult mice are required to delineate these possibilities.

\section{ESE-1 IN AIRWAY INFLAMMATION}

Our research group has previously shown that ESE-1 expression is upregulated in human bronchial airway epithelial cell lines after treatment with the proinflammatory cytokines, interleukin- $1 \beta$ (IL-1 $\beta$ ) and tumor necrosis factor$\alpha(\mathrm{TNF}-\alpha) \cdot{ }^{44}$ Furthermore, this cytokine-induced expression of ESE-1 is mediated by activation of the transcription factor, nuclear factor $-\kappa \mathrm{B}(\mathrm{NF}-\kappa \mathrm{B})$, and after thorough characterization of the ESE-1 gene promoter, the NF- $\kappa \mathrm{B}$ binding sequences that are required for this upregulation of ESE-1 expression were identified. ${ }^{44}$ Indeed, others have also previously shown the presence of TATA and CCAAT boxes, as well as potential binding sites for various ETS factors and $\mathrm{NF}-\kappa \mathrm{B}$ within the promoter region of the ESE-1 gene. ${ }^{45}$ In addition, we had also demonstrated that ESE-1 upregulates the expression of another member of the ESE subfamily of ETS transcription factors, ESE-3, and downregulates its own induction by cytokines, IL- $1 \beta$ and TNF- $\alpha .{ }^{44}$ Lastly, we found there to be reduced protein levels of the proinflammatory cytokine, IL-6, in the bronchoalveolar lavage fluid, lung tissue extract, and serum of Elf3-/- mice as compared with their wild-type littermates after intranasal instillation of lipopolysaccharide (LPS), ${ }^{44}$ suggesting a possible role for Elf3 in the regulation of IL- 6 expression within the setting of airway inflammation. Interestingly, in cultured primary airway epithelial cells, ESE-1 has also been reported to transactivate the promoter of the human lysozyme gene, which is an essential component of innate immune defense in the lung epithelia. ${ }^{46}$

Since Elf3-/ - mice had shown impairment in IL-6 production upon exposure to LPS and IL-6 is a key cytokine involved in $\mathrm{T}_{\mathrm{H}} 17$ differentiation, we also examined a potential role of Elf3 in regulating pulmonary inflammation using an airway inflammation model that is known to be dependent on $\mathrm{T}_{\mathrm{H}} 17$ response. ${ }^{47}$ Upon epicutaneous sensitization with the antigen, ovalbumin (OVA), followed by subsequent intranasal airway challenge with OVA, we have recently found that Elf3-/- mice mount an impaired $\mathrm{T}_{\mathrm{H}} 17$ response. ${ }^{47}$ Surprisingly, higher $\mathrm{T}_{\mathrm{H}} 2$ antibody titers along with a more severe extent of airway inflammation was observed in Elf3-/- mice as compared with their wild-type littermates. ${ }^{47}$ As these findings were likely due to an exaggerated $\mathrm{T}_{\mathrm{H}} 2$ response in Elf3-I- mice, we also investigated a possible involvement of Elf3 in $\mathrm{T}_{\mathrm{H}} 2$-driven allergic airway inflammation. ${ }^{47}$ Using a model of intraperitoneal sensitization with OVA followed by airway OVA challenge, we found that Elf3 $-1-$ mice did indeed mount an exaggerated $\mathrm{T}_{\mathrm{H}} 2$ response. ${ }^{47}$ Further analysis revealed that although Elf3-/$\mathrm{T}$ cells were normal, Elf3-/- dendritic cells (DCs) underwent hypermaturation and were impaired in the production of the $\mathrm{T}_{\mathrm{H}} 1$-inducing cytokine, IL-12, and the $\mathrm{T}_{\mathrm{H}} 17$-inducing cytokine, IL-6, which accounted for these exaggerated $\mathrm{T}_{\mathrm{H}} 2$ and impaired $\mathrm{T}_{\mathrm{H}} 17$ responses. ${ }^{47}$ In addition, although the regulation of genes encoding for $\mathrm{T}_{\mathrm{H}}$ 2-polarizing cytokines was normal in Elf3-/- airway epithelial cells, IL-6 production was markedly reduced. ${ }^{47}$ Taken together, these findings 
identify a key role for Elf3 in regulating allergic airway inflammation by controlling DC-driven T-cell differentiation in mice. Thus, human ESE-1 may be an important factor involved in regulating the development of $\mathrm{T}_{\mathrm{H}} 2$ - and $\mathrm{T}_{\mathrm{H}} 17$ dependent diseases, such as allergy and asthma. However, the extent of contribution from epithelial cells in these pathological processes is not completely clear yet and requires further investigation. Further studies aimed at examining a potential connection between polymorphisms within the $E S E-1$ gene and susceptibility to developing various airway inflammatory diseases, such as asthma and cystic fibrosis, are also needed to delineate these possibilities.

\section{ESE-1 IN MAMMARY GLAND DEVELOPMENT AND BREAST CANCER}

In situ expression analysis in human mammary gland has shown that ESE-1 is expressed specifically in the epithelial cells of the ductules and lobular structures. ${ }^{48}$ Also, ESE-1 has been reported to positively regulate transcription of the whey acidic protein $(W A P)$ gene in mammary epithelial cells, independently of lactogenic hormone treatment. ${ }^{48} W A P$ is one of the major milk proteins produced by mammary epithelial cells during pregnancy and lactation. Interestingly, Elf3 mRNA levels increase within the mammary gland epithelium during murine pregnancy and early lactation. ${ }^{49}$ This suggests that ESE-1 may function to control processes related to cellular proliferation and differentiation as the mammary gland undergoes extensive epithelial cell proliferation along with subsequent differentiation and milk protein synthesis during pregnancy and lactation. In addition, the fact that murine Elf3 is also induced during involution of the mammary gland epithelium after weaning suggests that ESE-1 may also play a role in the regulation of apoptotic pathways as mammary alveolar structures collapse and the secretory epithelial cells are removed during the apoptotic and remodeling phases of glandular involution. ${ }^{49}$ Further studies aimed at investigating the specific function of ESE-1 in both mammary gland development and involution are required to identify the exact role of this transcription factor in regulating these important physiological processes.

It is important to point out the fact that benign, nontransformed mammary epithelial cells, cell lines, and breast tissues express very low levels of ESE-1 protein. ${ }^{19}$ However, transient nuclear ESE-1 expression in MCF-10A/MCF-12A benign, non-transformed mammary epithelial cells results in apoptosis $^{19}$ and/or decreased cell proliferation, ${ }^{50}$ whereas similar nuclear ESE-1 expression in fully transformed mammary epithelial cells fails to induce apoptosis. ${ }^{19}$ Although it was previously presumed that many ETS factors transform mammary epithelial cells via their nuclear transcriptional functions, it has been shown that ESE-1 protein is localized within the cytoplasm in human breast cancer cells and that initiation of transformation of benign MCF-12A cells by stable expression of ESE-1 is mediated by the SAR domain of ESE-1, which acts in the cytoplasm via an unknown non- nuclear and non-transcriptional mechanism. ${ }^{19}$ Importantly, the nuclear localization of ESE-1 protein maintains the transformed phenotype of fully transformed mammary epithelial cells. ${ }^{51}$ Others have reported that the signaling kinase, Pak1, interacts with and selectively phosphorylates ESE-1 at serine 207, which is located within the SAR domain. ${ }^{20}$ It was further demonstrated that ESE-1 is a labile protein, which readily undergoes ubiquitin-dependent proteolysis by interacting with the F-box-binding protein, $\beta$-transducin repeat-containing protein, and that the stability and transforming potential of ESE-1 can be enhanced via this phosphorylation-dependent regulation by Pak $1 .{ }^{20}$ Taken together, these studies have provided valuable knowledge towards understanding the molecular mechanisms of ESE-1-mediated transformation; however, the exact cytoplasmic- and Pak1-dependent mechanism by which ESE-1 initiates transformation in mammary epithelial cells still remains to be determined. Interestingly, others have demonstrated that ESE-1 expression alone can induce a transformed and in vitro metastatic phenotype along with functional and morphological epithelial-to-mesenchymal transition in otherwise normal MCF-12A human breast epithelial cells. ${ }^{52}$ Furthermore, specific knockdown of endogenous ESE-1 in the human breast carcinoma ZR-75-1 and MCF-7 cell lines decreased cellular proliferation, colony formation, and anchorage-independent growth. ${ }^{51}$ Collectively, these findings suggest that ESE-1 plays a key role in maintaining the transformed phenotype by controlling cell proliferation in human breast cancer cells, thus providing a potentially novel target for future breast cancer therapy.

ESE-1 is located at human chromosome 1q32.1 in a region known to be amplified in $50 \%$ of early breast cancers, ${ }^{53}$ and ESE-1 mRNA is overexpressed at an early stage of human breast cancer development known as ductal carcinoma in situ. ${ }^{54}$ In addition, the presence of both fully spliced and partially unspliced forms of ESE-1 mRNA has been detected in human breast cancer cell lines and breast cancer tissues. ${ }^{55}$ Moreover, higher levels of ESE-1 mRNA and protein expression were detected in breast cancer cells than in normal breast epithelial cells, and an overexpression of ESE-1 has been observed in primary breast tumor specimens as compared to normal mammary tissues. ${ }^{56}$ ESE-1 expression is also upregulated in a subset of breast tumors and breast cancer-derived cell lines that express high levels of the Her2/Neu proto-oncogene, which is also known as erythroblastic leukemia viral oncogene homolog 2 (ErbB-2). ${ }^{54}$ Transient reporter assays using the ESE-1 promoter have shown that ESE-1 transcription is regulated by ErbB-2 receptor signaling in epithelial breast cancer cells, where expression of ErbB-2 upregulates ESE-1 promoter activity, while inhibition of ErbB-2 or its downstream signaling pathways decrease both ESE-1 promoter activity and endogenous ESE-1 protein levels. ${ }^{57}$ Thus, these findings identified the ESE-1 promoter as a potential transcriptional target of ErbB-2, and indicate ESE-1 expression as a downstream mediator of ErbB-2 signaling and ErbB-2-induced 
gene expression in the context of breast tumorigenesis. ${ }^{57}$ It has also been shown that ESE-1 is able to activate several malignancy-associated gene promoters, including the Her2/Neu (ErbB-2) promoter, and that ESE-1 expression is required for cellular survival of both non-transformed MCF-12A and transformed T47D human mammary epithelial cells in colony formation assays. ${ }^{58}$ Therefore, a positive feedback loop with both ErbB-2 and ESE-1 is believed to occur based on the observations of increased ESE-1 expression in response to Her2/Neu receptor activation and ESE-1 subsequently binding and activating the Her2/Neu gene promoter. Moreover, it has also been reported that ESE-1 and ErbB-2 can cooperate to confer an invasive phenotype in human mammary epithelial cells. ${ }^{50}$ In contrast, overexpression of ESE-1 in human breast cancer cells has been shown to induce endogenous TGF- $\beta$ RII expression, resulting in restitution of the TGF- $\beta$ signaling pathway along with a significant reduction in resistance to the growth inhibitory effects of TGF- $\beta .^{32}$

\section{ESE-1 IN TERMINAL DIFFERENTIATION OF THE EPIDERMAL SKIN EPITHELIUM}

An induction of ESE-1 expression has been reported to occur during terminal differentiation of the skin epidermis and in a primary human keratinocyte differentiation system. ${ }^{17}$ Moreover, transient reporter assays have shown that ESE-1 activates the expression of genes critical for terminal differentiation of epidermal keratinocytes, such as small prolinerich protein $2 \mathrm{~A}$ (SPRR2A), ${ }^{17}$ transglutaminase- $3,{ }^{59}$ and profilaggrin. ${ }^{59}$ It has also been demonstrated that Skn-1a, a specific isoform of the transcription factor Skn-1, interacts and functionally cooperates with ESE- 1 in transcriptional activation of the SPRR2A promoter in a human keratinocyte cell line. ${ }^{60}$ The human keratin 4 gene plays an important role in early differentiation of the esophageal squamous epithelium, and ESE-1 was also reported to suppress basal keratin 4 promoter activity and simultaneously activate the late differentiation linked SPRR2A promoter in both esophageal and cervical cancer epithelial cell lines. ${ }^{61}$ Interestingly, both ESE-1 and AP-1 have been shown to regulate cooperatively the expression of another human keratinocyte terminal differentiation marker, SPRR1A, at the proximal promoter region of the gene. ${ }^{62}$ In addition, the promoter region of the human SPRR3 gene contains multiple regulatory elements involved in keratinocyte differentiation-specific expression, including a high-affinity ETS binding site bound by ESE-1. ${ }^{63}$ Collectively, the findings obtained from the aforementioned studies suggest that ESE-1 plays an important role in regulating terminal differentiation of the epidermal epithelium; however, further studies are required to understand the exact role of ESE-1 in this important physiological process.

\section{ESE-1 IN THE CORNEAL AND RETINAL EPITHELIUM OF THE EYE}

ESE-1 has been shown to be upregulated upon differentiation in the embryonic/postnatal and adult mouse corneal epi- thelium and in immortalized human corneal epithelial cells, and this upregulation correlated with increased expression of K12 keratin, which is a marker for differentiated corneal epithelial cells. ${ }^{64}$ High levels of Elf3 expression have also been reported to occur within the retinal pigment epithelium (RPE) of the retina in rats. ${ }^{65}$ ESE- 1 has also been shown to be expressed in the human RPE cell lines, D407 and hTERTRPE1. ${ }^{65}$ Moreover, ESE-1 was shown to upregulate the promoter of an important ocular gene, tissue inhibitor of metalloproteinase 3 (TIMP3). ${ }^{65}$ Thus, the specific expression of ESE-1 in the RPE may reflect an important role for this transcription factor in retinal function. Furthermore, the regulation of TIMP3 expression by ESE-1 may have implications for degenerative retinal diseases, such as agerelated macular degeneration. Future experiments aimed at discovering the precise role of ESE-1 within both the corneal epithelium and RPE of the eye are required to clarify these possibilities.

\section{ESE-1 IN NON-EPITHELIAL CELLS IN THE SETTING OF INFLAMMATION}

While ESE-1 is believed to be expressed exclusively in epithelial cells, this may only be the case under basal conditions as numerous studies have shown that expression of ESE-1 can be induced by the proinflammatory cytokines, IL-1 $\beta$ and TNF- $\alpha$, and LPS in non-epithelial cells, such as synovial fibroblasts, ${ }^{66,67}$ chondrocytes,${ }^{66,68}$ osteoblasts, ${ }^{66}$ monocytes/ macrophages, ${ }^{66,69,70}$ vascular smooth muscle cells, ${ }^{69,71}$ and endothelial cells. ${ }^{69,71,72}$ More specifically, it has been shown that this induction relies on the translocation of the NF- $\kappa \mathrm{B}$ family members p50 and p 65 to the nucleus and subsequent transactivation of the ESE-1 promoter by a high-affinity NF- $\kappa$ B binding site. ${ }^{66}$ Furthermore, the expression of ESE-1 has also been observed in cells of the synovial lining layer and in some mononuclear and endothelial cells in inflamed synovial tissues from patients with rheumatoid arthritis and osteoarthritis. ${ }^{66}$ Collectively, these findings suggest that ESE-1 may play a role in mediating some of the effects of proinflammatory stimuli in various cells at the sites of inflammation. It has also been demonstrated that ESE-1 can specifically bind and transactivate the promoter of the gene encoding for angiopoietin-1 (Ang-1), which is an important angiogenic growth factor that promotes the chemotaxis of endothelial cells and facilitates the maturation of new blood vessels during the process of angiogenesis in various inflammatory responses. ${ }^{67}$ Moreover, ESE- 1 and Ang-1 are induced in synovial fibroblasts in response to inflammatory cytokines, with ESE-1 induction slightly preceding that of Ang-1, and both ESE-1 and Ang-1 exhibit a similar and strong expression pattern in the synovium of patients with rheumatoid arthritis. ${ }^{67}$ Taken together, these findings imply that ESE-1 may also function as a transcriptional mediator of angiogenesis in the setting of inflammation. Interestingly, an induction of ESE-1 by IL- $1 \beta$ was found to occur in human chondrocytes, with ESE-1 functioning as a potent 
transcriptional suppressor of promoter activity for the type II collagen (COL2A1) gene and accounting for the sustained, NF- $\kappa$ B-dependent inhibition of COL2A1 expression by IL-1 $\beta .^{68}$ Moreover, intracellular staining for ESE-1 was observed in chondrocytes from patients with osteoarthritis, but not in normal cartilage, suggesting a fundamental role for ESE-1 in cartilage degeneration and suppression of repair. ${ }^{68}$

Substantial evidence for the involvement of ESE-1 in vascular inflammation has also been provided from many different studies. For instance, ESE-1 has been shown to interact with the p50 subunit of NF- $\kappa \mathrm{B}$ during the regulation of transcription of the inducible nitric-oxide synthase (NOS2) gene. ${ }^{69}$ Furthermore, strong expression of ESE-1 has been observed within the vascular smooth muscle and endothelium in a mouse model of endotoxemia, which is associated with acute vascular inflammation. ${ }^{69}$ Others have reported that ESE-1 binds to and activates the promoter of the cyclooxygenase-2 (COX-2) gene in monocytes/macrophages in response to LPS. ${ }^{70}$ As COX-2 is a key enzyme involved in the production of prostaglandins that are major inflammatory agents, neutralization of COX-2 is a potentially important aim for designing many anti-inflammatory drugs, and as an activator of COX-2 induction, ESE-1 is a potential target for such therapeutics as well. ${ }^{70}$ Other proteins, including the transcriptional co-activators p300 and cAMPresponse element-binding-binding protein, have been shown to interact with ESE-1 and enhance its transcriptional activity in vascular endothelial cells. ${ }^{71}$ In contrast, the Ku proteins, Ku70 and Ku86, which are involved in the repair of DNA damage, have been reported to bind to the DNA-binding domain of ESE-1 and negatively regulate its transcriptional activity. ${ }^{71}$ In vivo evidence for an involvement of ESE- 1 in vascular inflammation has also been provided in an angiotensin II (Ang II)-induced model of vascular inflammation and remodeling, where Elf3-/- mice have been observed to exhibit increased inflammatory cell infiltration into the aortic vessel wall, intimal-medial thickening, and perivascular fibrosis of the aorta, as well as elevated systolic blood pressure as compared to the wild-type littermate controls. ${ }^{72}$ Interestingly, reduced expression of NOS2 was also observed in Elf3-I- mice infused with Ang II, suggesting that maintenance of NOS2 expression may be a mechanism by which ESE-1 offers vascular protection. ${ }^{72}$ Collectively, these findings provide valuable insight with regard to the involvement of ESE-1 in vascular dysfunction in the context of inflammation; however, further studies are required to confirm the putative vascular protective effects of ESE-1-mediated NO release in the Ang II-dependent model of vascular inflammation.

\section{CONCLUDING REMARKS}

In conclusion, the studies discussed in this review have provided ample experimental evidence that the epitheliumspecific ETS transcription factor, ESE-1, is involved in regulating a variety of pathophysiological processes. Although the expression of ESE-1 was initially believed to occur exclusively in epithelial cells, many experiments have now proven that ESE-1 expression can be induced by inflammatory stimuli in various cell types of non-epithelial origin. Thus, ESE-1 is a multifaceted transcription factor that participates in many biological processes, mainly including controlling epithelial cell differentiation by regulating TGF- $\beta$ RII expression during both intestinal development and lung regeneration; regulating DC-driven T-cell differentiation as well as IL-6 expression during allergic airway inflammation; and mediating the effects of proinflammatory stimuli in the setting of synovial joint tissue and vascular inflammation. Moreover, it seems quite possible that distinct patterns of expression of its target genes may account for the divergent roles of ESE-1 in tissue stasis and inflammation. Given the similarities of the physiological process of development with the pathological process of neoplasia as well as the role of inflammatory processes in influencing cancer progression, it is also quite possible that in certain cancer states (eg breast and/or lung cancer), ESE-1 may play homeostatic roles akin to its role in regulating both development and inflammation. Although previous studies have identified a role of ESE-1 in the aforementioned processes, further studies are needed to fully understand the exact underlying molecular mechanisms. Furthermore, as there is substantial evidence for the involvement of ESE-1 in regulating a diverse range of pathological processes overall, there is a high likelihood for this transcription factor to be a candidate susceptibility gene for various disorders.

\section{ACKNOWLEDGEMENTS}

This work was supported in part by operating Grants from the Canadian Institutes of Health Research No. MOP-97779 and from the Canadian Cystic Fibrosis Foundation to JH. JRO is a recipient of the Canadian Institutes of Health Research Canada Graduate Scholarships Doctoral Award.

\section{DISCLOSURE/CONFLICT OF INTEREST}

The authors declare no conflict of interest.

1. Wasylyk B, Hahn SL, Giovane A. The Ets family of transcription factors. Eur J Biochem 1993;211:7-18.

2. Oikawa T, Yamada T. Molecular biology of the Ets family of transcription factors. Gene 2003;303:11-34.

3. Wei GH, Badis G, Berger MF, et al. Genome-wide analysis of ETS-family DNA-binding in vitro and in vivo. EMBO J 2010;29:2147-2160.

4. Gutierrez-Hartmann A, Duval DL, Bradford AP. ETS transcription factors in endocrine systems. Trends Endocrinol Metab 2007;18:150-158.

5. Kageyama S, Liu H, Nagata M, et al. The role of ETS transcription factors in transcription and development of mouse preimplantation embryos. Biochem Biophys Res Commun 2006;344:675-679.

6. Jedlicka $P$, Gutierrez-Hartmann A. Ets transcription factors in intestinal morphogenesis, homeostasis and disease. Histol Histopathol 2008;23: 1417-1424.

7. Lincoln II DW, Bove K. The transcription factor Ets-1 in breast cancer. Front Biosci 2005;10:506-511.

8. Turner DP, Findlay VJ, Moussa O, et al. Defining ETS transcription regulatory networks and their contribution to breast cancer progression. J Cell Biochem 2007;102:549-559.

9. Turner DP, Watson DK. ETS transcription factors: oncogenes and tumor suppressor genes as therapeutic targets for prostate cancer. Expert Rev Anticancer Ther 2008;8:33-42. 
10. Lacorazza HD, Nimer SD. The emerging role of the myeloid Elf-1 like transcription factor in hematopoiesis. Blood Cells Mol Dis 2003;31: 342-350.

11. Gupta P, Gurudutta GU, Saluja D, et al. PU.1 and partners: regulation of haematopoietic stem cell fate in normal and malignant haematopoiesis. J Cell Mol Med 2009;13:4349-4363.

12. Lelievre $\mathrm{E}$, Lionneton $\mathrm{F}$, Soncin $\mathrm{F}$, et al. The Ets family contains transcriptional activators and repressors involved in angiogenesis. Int J Biochem Cell Biol 2001;33:391-407.

13. Dejana E, Taddei A, Randi AM. Foxs and Ets in the transcriptional regulation of endothelial cell differentiation and angiogenesis. Biochim Biophys Acta 2007;1775:298-312.

14. Randi AM, Sperone A, Dryden NH, et al. Regulation of angiogenesis by ETS transcription factors. Biochem Soc Trans 2009;37:1248-1253.

15. Oettgen P. Regulation of vascular inflammation and remodeling by ETS factors. Circ Res 2006;99:1159-1166.

16. Tymms MJ, Ng AY, Thomas RS, et al. A novel epithelial-expressed ETS gene, ELF3: human and murine CDNA sequences, murine genomic organization, human mapping to 1q32.2 and expression in tissues and cancer. Oncogene 1997;15:2449-2462.

17. Oettgen $P$, Alani RM, Barcinski MA, et al. Isolation and characterization of a novel epithelium-specific transcription factor, ESE-1, a member of the ets family. Mol Cell Biol 1997;17:4419-4433.

18. Feldman RJ, Sementchenko VI, Watson DK. The epithelial-specific Ets factors occupy a unique position in defining epithelial proliferation, differentiation and carcinogenesis. Anticancer Res 2003;23:2125-2131.

19. Prescott JD, Koto KS, Singh M, et al. The ETS transcription factor ESE-1 transforms MCF-12A human mammary epithelial cells via a novel cytoplasmic mechanism. Mol Cell Biol 2004;24:5548-5564.

20. Manavathi B, Rayala SK, Kumar R. Phosphorylation-dependent regulation of stability and transforming potential of ETS transcriptional factor ESE-1 by p21-activated kinase 1. J Biol Chem 2007;282: 19820-19830.

21. Zhou J, Ng AY, Tymms MJ, et al. A novel transcription factor, ELF5, belongs to the ELF subfamily of ETS genes and maps to human chromosome $11 \mathrm{p} 13-15$, a region subject to $\mathrm{LOH}$ and rearrangement in human carcinoma cell lines. Oncogene 1998;17:2719-2732.

22. Oettgen $P$, Kas $K$, Dube $A$, et al. Characterization of ESE-2, a novel ESE1-related Ets transcription factor that is restricted to glandular epithelium and differentiated keratinocytes. J Biol Chem 1999;274: 29439-29452.

23. Kas K, Finger E, Grall F, et al. ESE-3, a novel member of an epitheliumspecific ets transcription factor subfamily, demonstrates different target gene specificity from ESE-1. J Biol Chem 2000;275:2986-2998.

24. Oettgen $P$, Finger $E$, Sun $Z$, et al. PDEF, a novel prostate epitheliumspecific ets transcription factor, interacts with the androgen receptor and activates prostate-specific antigen gene expression. J Biol Chem 2000;275:1216-1225.

25. Ng AY, Waring $P$, Ristevski $S$, et al. Inactivation of the transcription factor Elf3 in mice results in dysmorphogenesis and altered differentiation of intestinal epithelium. Gastroenterology 2002;122: $1455-1466$.

26. Zorn AM, Wells JM. Molecular basis of vertebrate endoderm development. Int Rev Cytol 2007;259:49-111.

27. Albert $\mathrm{M}$, Peters $\mathrm{AH}$. Genetic and epigenetic control of early mouse development. Curr Opin Genet Dev 2009;19:113-121.

28. Takahashi K, Yamanaka S. Induction of pluripotent stem cells from mouse embryonic and adult fibroblast cultures by defined factors. Cell 2006;126:663-676.

29. Wernig $M$, Meissner $A$, Foreman $R$, et al. In vitro reprogramming of fibroblasts into a pluripotent ES-cell-like state. Nature 2007;448: 318-324.

30. Flentjar N, Chu PY, Ng AY, et al. TGF-betaRll rescues development of small intestinal epithelial cells in Elf3-deficient mice. Gastroenterology 2007;132:1410-1419.

31. Choi SG, Yi Y, Kim YS, et al. A novel ets-related transcription factor, ERT/ ESX/ESE-1, regulates expression of the transforming growth factorbeta type II receptor. J Biol Chem 1998;273:110-117.

32. Chang J, Lee C, Hahm KB, et al. Over-expression of ERT(ESX/ESE-1/ ELF3), an ets-related transcription factor, induces endogenous TGFbeta type II receptor expression and restores the TGF-beta signaling pathway in Hs578t human breast cancer cells. Oncogene 2000;19: $151-154$
33. Kim JH, Wilder PJ, Hou J, et al. Activation of the murine type II transforming growth factor-beta receptor gene: up-regulation and function of the transcription factor Elf-3/Ert/Esx/Ese-1. J Biol Chem 2002;277:17520-17530.

34. Lee HJ, Chang JH, Kim YS, et al. Effect of ets-related transcription factor (ERT) on transforming growth factor (TGF)-beta type II receptor gene expression in human cancer cell lines. J Exp Clin Cancer Res 2003;22: 477-480.

35. Kopp JL, Wilder PJ, Desler $\mathrm{M}$, et al. Unique and selective effects of five Ets family members, Elf3, Ets1, Ets2, PEA3, and PU.1, on the promoter of the type II transforming growth factor-beta receptor gene. J Biol Chem 2004;279:19407-19420.

36. Agarkar VB, Babayeva ND, Rizzino A, et al. Preliminary crystallographic analysis of mouse Elf3 C-terminal DNA-binding domain in complex with type II TGF-beta receptor promoter DNA. Acta Crystallogr Sect F 2009;65:1261-1263.

37. Agarkar VB, Babayeva ND, Wilder PJ, et al. Crystal structure of mouse Elf3 C-terminal DNA-binding domain in complex with type II TGF-beta receptor promoter DNA. J Mol Biol 2010;397:278-289.

38. Park SH, Kim YS, Park BK, et al. Sequence-specific enhancer binding protein is responsible for the differential expression of ERT/ESX/ELF-3/ ESE-1/jen gene in human gastric cancer cell lines: implication for the loss of TGF-beta type II receptor expression. Oncogene 2001;20: $1235-1245$.

39. Kwon JH, Keates S, Simeonidis S, et al. ESE-1, an enterocyte-specific Ets transcription factor, regulates MIP-3alpha gene expression in Caco-2 human colonic epithelial cells. J Biol Chem 2003;278:875-884.

40. Kwon MC, Koo BK, Kim YY, et al. Essential role of CR6-interacting factor 1 (Crif1) in E74-like factor 3 (ELF3)-mediated intestinal development. J Biol Chem 2009;284:33634-33641.

41. Reddy SP, Vuong $\mathrm{H}$, Adiseshaiah P. Interplay between proximal and distal promoter elements is required for squamous differentiation marker induction in the bronchial epithelium: role for ESE-1, Sp1, and AP-1 proteins. J Biol Chem 2003;278:21378-21387.

42. Oliver JR, Kushwah $\mathrm{R}, \mathrm{Wu} \mathrm{J}$, et al. Elf3 plays a role in regulating bronchiolar epithelial repair kinetics following Clara cell-specific injury. Lab Invest 2011;91:1514-1529.

43. Atkinson JJ, Toennies HM, Holmbeck K, et al. Membrane type 1 matrix metalloproteinase is necessary for distal airway epithelial repair and keratinocyte growth factor receptor expression after acute injury. Am J Physiol Lung Cell Mol Physiol 2007;293:L600-L610.

44. Wu J, Duan $\mathrm{R}, \mathrm{Cao} \mathrm{H}$, et al. Regulation of epithelium-specific Ets-like factors ESE-1 and ESE-3 in airway epithelial cells: potential roles in airway inflammation. Cell Res 2008;18:649-663.

45. Oettgen $P$, Barcinski $M$, Boltax J, et al. Genomic organization of the human ELF3 (ESE-1/ESX) gene, a member of the Ets transcription factor family, and identification of a functional promoter. Genomics 1999;55:358-362.

46. Lei W, Jaramillo RJ, Harrod KS. Transactivation of lung lysozyme expression by Ets family member ESE-1. Am J Physiol Lung Cell Mol Physiol 2007;293:L1359-L1368.

47. Kushwah $\mathrm{R}$, Oliver JR, Wu J, et al. Elf3 regulates allergic airway inflammation by controlling dendritic cell-driven $\mathrm{T}$ cell differentiation. $\mathrm{J}$ Immunol 2011;187:4639-4653.

48. Thomas RS, Ng AN, Zhou J, et al. The Elf group of Ets-related transcription factors. ELF3 and ELF5. Adv Exp Med Biol 2000;480: 123-128.

49. Neve R, Chang CH, Scott GK, et al. The epithelium-specific ets transcription factor ESX is associated with mammary gland development and involution. FASEB J 1998;12:1541-1550.

50. Coppe JP, Amend C, Semeiks J, et al. ERBB receptor regulation of ESX/ ELF3 promotes invasion in breast epithelial cells. Open Cancer J 2010;3:89-100.

51. Walker DM, Poczobutt JM, Gonzales MS, et al. ESE-1 is required to maintain the transformed phenotype of MCF-7 and ZR-75-1 human breast cancer cells. Open Cancer J 2010;3:77-88.

52. Schedin PJ, Eckel-Mahan KL, McDaniel SM, et al. ESX induces transformation and functional epithelial to mesenchymal transition in MCF-12A mammary epithelial cells. Oncogene 2004;23: 1766-1779.

53. Oettgen $\mathrm{P}$, Carter KC, Augustus $\mathrm{M}$, et al. The novel epithelial-specific Ets transcription factor gene ESX maps to human chromosome 1q32.1. Genomics 1997;45:456-457. 
54. Chang $\mathrm{CH}$, Scott GK, Kuo WL, et al. ESX: a structurally unique Ets overexpressed early during human breast tumorigenesis. Oncogene 1997;14:1617-1622.

55. Kaplan MH, Wang XP, Xu HP, et al. Partially unspliced and fully spliced ELF3 mRNA, including a new Alu element in human breast cancer. Breast Cancer Res Treat 2004;83:171-187.

56. He J, Pan Y, Hu J, et al. Profile of Ets gene expression in human breast carcinoma. Cancer Biol Ther 2007;6:76-82.

57. Neve RM, Ylstra B, Chang $\mathrm{CH}$, et al. ErbB2 activation of ESX gene expression. Oncogene 2002;21:3934-3938.

58. Eckel KL, Tentler JJ, Cappetta GJ, et al. The epithelial-specific ETS transcription factor ESX/ESE-1/Elf-3 modulates breast cancerassociated gene expression. DNA Cell Biol 2003;22:79-94.

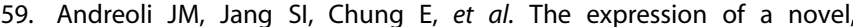
epithelium-specific ets transcription factor is restricted to the most differentiated layers in the epidermis. Nucleic Acids Res 1997;25: 4287-4295.

60. Cabral A, Fischer DF, Vermeij WP, et al. Distinct functional interactions of human Skn-1 isoforms with Ese-1 during keratinocyte terminal differentiation. J Biol Chem 2003;278:17792-17799.

61. Brembeck FH, Opitz OG, Libermann TA, et al. Dual function of the epithelial specific ets transcription factor, ELF3, in modulating differentiation. Oncogene 2000;19:1941-1949.

62. Sark MW, Fischer DF, de Meijer E, et al. AP-1 and ets transcription factors regulate the expression of the human SPRR1A keratinocyte terminal differentiation marker. J Biol Chem 1998;273 24683-24692.

63. Fischer DF, Sark MW, Lehtola MM, et al. Structure and evolution of the human SPRR3 gene: implications for function and regulation. Genomics 1999;55:88-99.
64. Yoshida $\mathrm{N}$, Yoshida $\mathrm{S}$, Araie $\mathrm{M}$, et al. Ets family transcription factor ESE1 is expressed in corneal epithelial cells and is involved in their differentiation. Mech Dev 2000;97:27-34.

65. Jobling Al, Fang Z, Koleski D, et al. Expression of the ETS transcription factor ELF3 in the retinal pigment epithelium. Invest Ophthalmol Vis Sci 2002;43:3530-3537.

66. Grall F, Gu X, Tan L, et al. Responses to the proinflammatory cytokines interleukin-1 and tumor necrosis factor alpha in cells derived from rheumatoid synovium and other joint tissues involve nuclear factor kappaB-mediated induction of the Ets transcription factor ESE-1. Arthritis Rheum 2003;48:1249-1260.

67. Brown C, Gaspar J, Pettit A, et al. ESE-1 is a novel transcriptional mediator of angiopoietin-1 expression in the setting of inflammation. J Biol Chem 2004;279:12794-12803.

68. Peng $H$, Tan L, Osaki M, et al. ESE-1 is a potent repressor of type II collagen gene (COL2A1) transcription in human chondrocytes. J Cell Physiol 2008;215:562-573.

69. Rudders S, Gaspar J, Madore R, et al. ESE-1 is a novel transcriptional mediator of inflammation that interacts with NF-kappa B to regulate the inducible nitric-oxide synthase gene. J Biol Chem 2001;276:3302-3309.

70. Grall FT, Prall WC, Wei W, et al. The Ets transcription factor ESE-1 mediates induction of the COX-2 gene by LPS in monocytes. FEBS J 2005;272:1676-1687.

71. Wang H, Fang R, Cho JY, et al. Positive and negative modulation of the transcriptional activity of the ETS factor ESE-1 through interaction with p300, CREB-binding protein, and Ku 70/86. J Biol Chem 2004;279: 25241-25250.

72. Zhan $Y$, Yuan $L$, Kondo $M$, et al. The counter-regulatory effects of ESE-1 during angiotensin II-mediated vascular inflammation and remodeling. Am J Hypertens 2010;23:1312-1317. 